\section{Major depression subtypes are differentially associated with migraine subtype, prevalence and severity}

(C) International Headache Society 2019 Article reuse guidelines: sagepub.com/journals-permissions DOI: $10.1177 / 0333102419884935$ journals.sagepub.com/home/cep

@SAGE

\author{
Claudia Pisanu ${ }^{1,2, *}$, Emma Lundin ${ }^{1, *}$, Martin Preisig ${ }^{3}$, \\ Mehdi Gholam-Rezaee ${ }^{3}$, Enrique Castelao ${ }^{3}$, Giorgio Pistis ${ }^{3}$, \\ Kathleen R Merikangas ${ }^{4}$, Jennifer Glaus ${ }^{4}$, Alessio Squassina ${ }^{2}$, \\ Maria Del Zompo ${ }^{2}$, Helgi B Schiöth ${ }^{1,5}$ and Jessica Mwinyi'
}

\begin{abstract}
Objective: Migraine and major depressive disorder show a high rate of comorbidity, but little is known about the associations between the subtypes of major depressive disorder and migraine. In this cross-sectional study we aimed at investigating a) the lifetime associations between the atypical, melancholic, combined and unspecified subtype of major depressive disorder and migraine with and without aura and b) the associations between major depressive disorder and its subtypes and the severity of migraine.

Methods: A total of 446 subjects with migraine (migraine without aura: $n=294$; migraine with aura: $n=152$ ) and $25 \mathrm{II}$ controls from the population-based CoLaus/PsyCoLaus study, Switzerland, were included. Associations between major depressive disorder subtypes and migraine characteristics were tested using binary logistic or linear regression.

Results: Melancholic, combined and unspecified major depressive disorder were associated with increased frequency of migraine with aura, whereas only melancholic major depressive disorder was associated with increased frequency of migraine without aura. Lifetime and unspecified major depressive disorder were associated with severe migraine intensity among subjects with migraine with aura but not migraine without aura, while combined major depressive disorder was associated with higher migraine frequency independently from migraine subtype.

Conclusion: This study suggests that melancholic but not atypical major depressive disorder is associated with migraine and migraine subtypes. Future studies exploring pathophysiological mechanisms shared between melancholic depression and migraine are warranted.
\end{abstract}

\title{
Keywords
}

Migraine with aura, migraine severity, major depressive disorder, melancholic depression

Date received: I February 2019; revised: 5 July 2019; 19 September 2019; accepted: I October 2019

\section{Introduction}

Migraine shows a high rate of comorbidity with major depressive disorder (MDD) (1). MDD lifetime prevalence differs between different populations and is estimated to be around $8-12 \%$, while the estimated heritability is about $40-50 \%$ (2). In patients with both migraine and depression, the exacerbation of one disease may increase the symptoms of the other illness (3). Each condition increases the relative risk for the other disease up to three times, yet the underlying mechanism leading to the high rate of coexistence is not yet well understood (4). Migraine patients with comorbid MDD
'Department of Neuroscience, University of Uppsala, Uppsala, Sweden ${ }^{2}$ Department of Biomedical Sciences, University of Cagliari, Cagliari, Italy ${ }^{3}$ Department of Psychiatry, Lausanne University Hospital, Prilly, Switzerland

${ }^{4}$ Genetic Epidemiology Research Branch, Intramural Research Program, National Institute of Mental Health, Bethesda, MD, USA

${ }^{5}$ Institute for Translational Medicine and Biotechnology, Sechenov First Moscow State Medical University, Moscow, Russia

*These authors contributed equally to this work.

\section{Corresponding author:}

Jessica Mwinyi, Department of Neuroscience, Division of Functional Pharmacology, BMC, Uppsala University Husargatan 3, 75I24 Uppsala, Sweden.

Email: jessica.mwinyi@neuro.uu.se 
are less likely to show a complete remission and have an increased risk of developing chronic migraine (5).

Major depression has a high degree of heterogeneity in terms of clinical characteristics, course and response to pharmacological treatment (6). It has been hypothesized that depression subtypes might be associated with different biological mechanisms (7). Melancholic depression, which is present in approximately $30 \%$ of depressed individuals (8), is characterized by anhedonia, psychomotor disturbances, interrupted sleep and loss of appetite (9). Biological correlates of melancholic depression include hypercortisolemia, leading to the hypothesis that this subtype might be related to dysfunction of the hypothalamic-pituitary-adrenal (HPA) axis. Conversely, atypical depression, which is present in $15-30 \%$ of depressed individuals (8), is characterized by increased appetite, weight gain and hypersomnia (9). This subtype has been suggested to be associated with metabolic syndrome and inflammation $(9,10)$.

The assessment of associations between depression and migraine subtypes may help clinicians to identify individuals with a particular risk of comorbid disorders and favor early treatment. In addition, the identification of depression and migraine subtypes that are frequently comorbid and associated with a higher degree of severity can help to further elucidate the mechanisms playing a role in migraine pathogenesis as well as to identify potential migraine subphenotypes that might benefit from tailored treatment strategies.

Although the relationship between depression and migraine is well known, to our knowledge no study has investigated the relationship between migraine and different depression subtypes. Based on this evidence, the aim of this study was to investigate a) the lifetime associations between the atypical, melancholic, combined and unspecified subtypes of MDD and migraine with (MWA) and without aura (MWOA) and $b$ ) the associations between MDD and its subtypes and the severity of migraine.

\section{Material and methods}

\section{Sample}

The present paper is based on the baseline evaluation of the population-based CoLaus|PsyCoLaus cohort study. The primary aim of this prospective cohort study was to evaluate mental disorders and cardiovascular risk factors in the community and to determine their associations. The sample was randomly selected from the 35 to 75 -year-old residents of the city of Lausanne, Switzerland, from 2003 to 2006 according to the civil register (11). Sixty-seven percent of the 35 to 66-year-old participants in the physical baseline exam $(n=5535)$ also accepted the psychiatric evaluation (12), resulting in a sample of 3719 participants with both somatic and psychiatric assessments. For the present analyses, we only included participants for whom information on migraine and MDD were available, leaving a total of 2957 subjects. The CoLaus|PsyCoLaus study was approved by the Institutional Ethics Committee of the University of Lausanne and informed written consent was obtained from all participants.

\section{Assessments}

Interviews at the psychiatric evaluation were carried out by trained master-level psychologists or by psychiatrists, whose training included rating tapes and supervised coratings. All interviews and assessments were reviewed by a senior psychologist afterwards. Diagnostic information on mental disorders including MDD was collected using the French version (13) of the semi-structured Diagnostic Interview for Genetic Studies (DIGS) (14). In participants endorsing either headache or migraine in the DIGS, the French version of the Diagnostic Interview for Headache Syndromes (DIHS) was used to collect additional data. This instrument was developed through an inter-site collaboration centered at the Genetic Epidemiology Research Unit of Yale University School of Medicine and translated into French by the research team that conducted the CoLaus ? PsyCoLaus study. The DIHS is a semi-structured interview that includes an open-ended section in which the participant describes each type of headache experienced, as well as a set of questions regarding symptoms, severity, frequency, duration and treatment. Migraine diagnoses were based on criteria of the International Headache Society (ICHD-II) (15). For the present study, we included data on severity indicators collected using three questions from the DIHS: a) Migraine intensity, rated by the participant as "light", "moderate" or "severe" (two participants who rated their migraine intensity as "light" were grouped together with those who rated their migraine intensity as "moderate"); b) frequency of attacks (expressed as average number of migraine days per month) and c) average duration of attacks (expressed in hours). The DIHS interview includes three questions to evaluate the average frequency of attacks: number of migraine days per week, month and year. In case a participant indicated the number of migraine days per week or year, an average number of migraine days per month was calculated by multiplying the number by 4 weeks or dividing it by 12 months, respectively. Similarly, in case a participant indicated the average duration of attacks in terms of days, the reported value was multiplied by 24 . A total of 446 subjects met the lifetime diagnostic criteria for migraine and had information on comorbidity with MDD. Information on migraine intensity, average frequency 
and duration was available for 445, 428 and 442 subjects, respectively.

For subjects meeting the DSM-IV criteria of MDD, the following subtypes were defined according to the lifetime occurrence of episodes (16): a) Melancholic type (MDD with at least one melancholic and no atypical episodes); b) atypical type (MDD with at least one atypical and no melancholic episodes; c) combined type (MDD with both atypical and melancholic features); and d) unspecified type (MDD with neither atypical nor melancholic episodes). The four-level migraine subtype variable was coded using four dummy variables, with participants with no diagnosis of MDD as the reference group.

Socioeconomic status (SES) was assessed using the Hollingshead scale, which includes information on education and professional position (17). SES was analyzed in quintiles using the following cut-off points: $1: \leq 20,2$ : 20-29, 3: 30-39, 4: 40-55, 5: $\geq 55$.

\section{Statistical analysis}

A study flow diagram is reported in Figure 1. Normal distribution of continuous variables was assessed using the Kolmogorov-Smirnov test. Pearson's Chi-squared test (for gender) or Mann-Whitney test (for age and
SES) were applied to analyze unadjusted differences between subjects with or without migraine/migraine subtypes.

Association between MDD subtypes and migraine. In order to evaluate the association with lifetime MDD, we applied a binary logistic regression model using migraine as the dependent variable and lifetime MDD or MDD subtypes as the independent variable, adjusting for sex, age and SES. Similarly, we applied two binary logistic regression models to assess the associations between MDD subtypes and the two migraine subtypes. In order to adjust for multiple testing (two migraine subtypes) the significance level was set to $<0.05 / 2=0.0025$.

Association between MDD subtypes and migraine severity. Within subjects with migraine we evaluated the associations between MDD subtypes and three indicators of migraine severity: Migraine intensity (light/moderate vs. severe intensity), migraine frequency (number of migraine days per month) and duration of an attack (hours). The associations between lifetime MDD or MDD subtypes and migraine intensity were evaluated using binary logistic regression models with either MDD or MDD subtypes as the independent variables

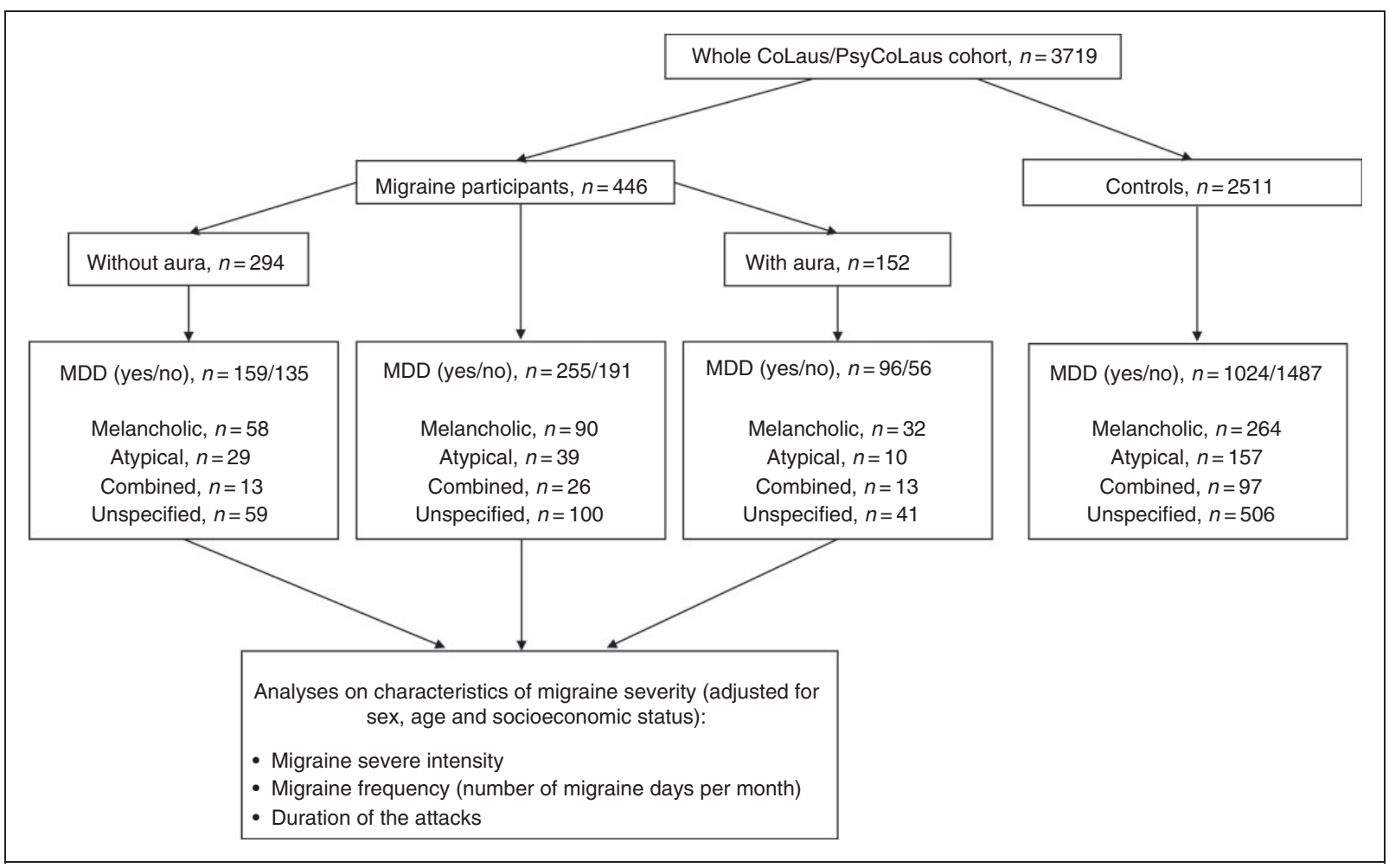

Figure I. Flow chart of the study. The figure shows the principal setup of the study with regards to number of subjects, migraine subtypes, lifetime major depressive disorder and subtypes and the principal statistical analyses on migraine severity. MDD: major depressive disorder. 
and migraine intensity as the dependent variable, with adjustment for sex, age and SES. The associations between MDD subtypes and the continuous indicators of migraine severity (migraine days per month and mean duration of attacks) were evaluated using linear regression models with MDD or MDD subtypes as the independent variables, log-transformed values of the indicator of migraine severity as the dependent variable, and with adjustment for sex, age, and SES. Normality and constant variance of residuals were checked through visual inspection of the residuals Q-Q plot and the scatter plot of standardized residuals versus the standardized predicted values, respectively. In order to assess the impact of migraine subtype on the associations between MDD/ MDD subtypes and migraine severity indicators, we first tested potential interactions between migraine subtype and MDD subtypes with respect to migraine severity indicators. If they showed a significant interaction $(p<0.05)$, a stratified model according to migraine subtype was performed, otherwise only the results of the model for the overall migraine diagnosis were presented. For analyses with the two migraine subtypes as the dependent variables, the level of significance was set to $p<0.025$ in order to adjust for multiple testing.

Statistical analyses were performed using SPSS v. 21 (IBM, Armonk, NY, USA).

\section{Results}

\section{Demographics and clinical characteristics in MWA and MWOA}

As shown in Table 1, individuals with migraine were more likely to be women regardless of the subtype. Individuals with any migraine and with the MWOA subtype were also younger and had lower SES than controls.

\section{Associations between MDD subtypes and migraine}

Lifetime MDD was significantly associated with migraine regardless of the subtype (Table 2). However, the association was stronger with the MWA than with the MWOA subtype. All MDD subtypes were associated with the overall category of migraine and all but atypical MDD were associated with MWA, whereas only melancholic MDD also revealed an association with MWOA.

\section{Associations between MDD and indicators of migraine severity}

The adjusted binary logistic regression models showed significant interactions between any $\operatorname{MDD}(p=0.002)$,

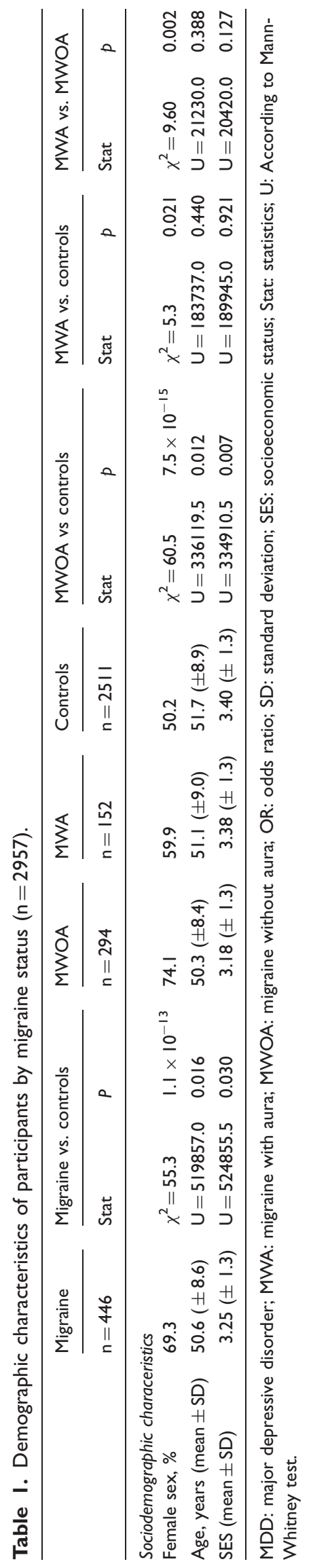




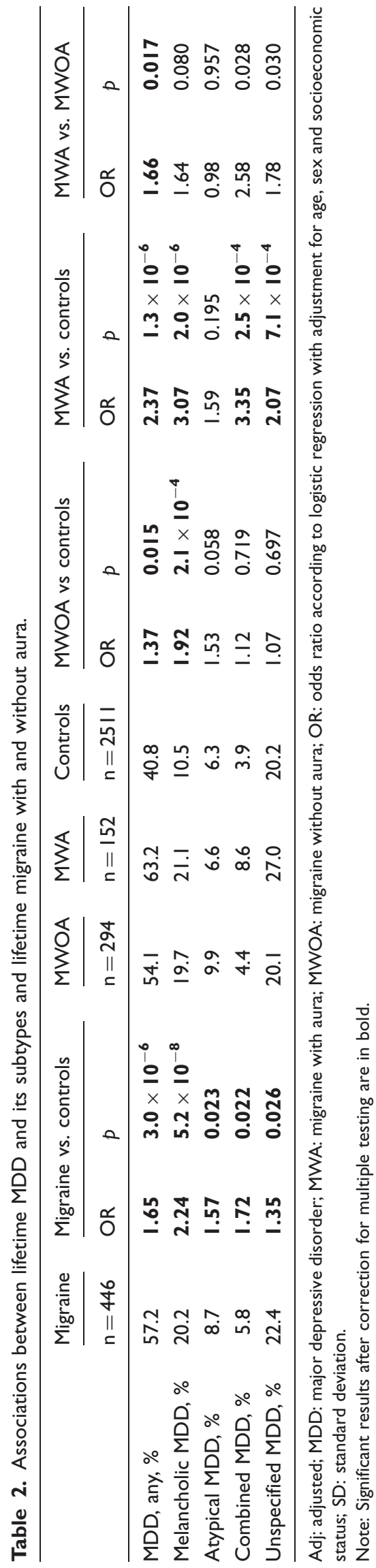

melancholic MDD $(p=0.047)$ and unspecified MDD $(p=0.002)$ and the migraine subtype with respect to "migraine intensity", indicating that the associations between any MDD or these MDD subtypes and migraine significantly differed according to the migraine subtype. Accordingly, only the results of stratified analyses are provided for these associations (Table 3). Conversely, as there was no evidence for interactions between atypical or combined MDD and the migraine subtype, only the associations between these MDD subtypes and overall migraine are presented. These analyses revealed that lifetime MDD and the unspecified subtype were significantly associated with higher migraine intensity in subjects with MWA but not in subjects with MWOA.

Regarding migraine frequency, the linear regression model showed a significant interaction between the melancholic MDD and the migraine subtype $(p=0.035)$. However, the stratified analysis showed that melancholic MDD was not associated with migraine frequency in the two migraine subtypes (Table 4). Migraine frequency was only associated with combined MDD.

Regarding migraine duration, the linear regression model showed significant interactions between any $\operatorname{MDD}(p=0.029)$, melancholic MDD $(p=0.044)$ and combined MDD $(p=0.038)$, indicating stronger associations in subjects with MWA. However, the stratified models did not provide evidence for significant associations between MDD or MDD subtypes and migraine duration (Table 5).

\section{Discussion}

This is the first report on comorbid associations between MDD subtypes, migraine subtypes and migraine severity indicators in the general population. Our most salient findings are that comorbid associations between MDD and migraine as well as associations between MDD and migraine severity indicators vary across both MDD and migraine subtypes. Moreover, all MDD subtypes were associated with the overall migraine diagnosis, showing strong comorbidity between MDD and migraine, and all but the atypical MDD subtype were associated with MWA, but only melancholic MDD was associated with MWOA. In contrast, few associations were found between MDD or its subtypes and migraine severity indicators.

It is well known that migraine and depression show a high rate of comorbidity. The association between these two conditions has been suggested to be bidirectional, with each disorder increasing the risk of onset of the other (1). Although a causal relationship cannot be precluded, the currently most accredited hypothesis is 


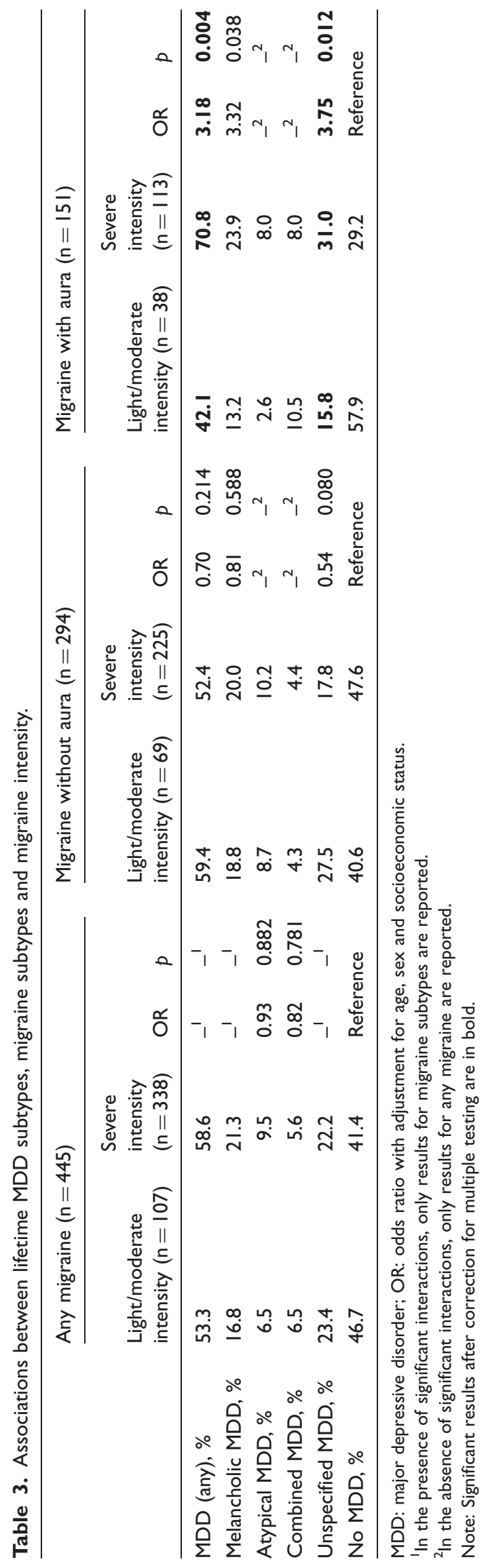

that shared disease mechanisms might underlie the comorbidity between these two conditions (18). This hypothesis is further supported by the observation that mood changes are amongst the most common premonitory symptoms in patients with migraine (19) as well as by the fact that the tricyclic antidepressant (TCA) amitriptyline is indicated in migraine prevention. Notably, while TCAs are effective in melancholic depression, patients with atypical depression more often show a poor response to these drugs (20).

There is a paucity of studies investigating the putative shared pathophysiological mechanisms between migraine and depression and, specifically, depression subtypes. Hippocampal dysfunction has been suggested to play a role in the pathogenesis of both diseases $(21,22)$. Being this region implicated in the inhibitory control of HPA-axis response to stress, hippocampal dysfunction could account for the association between MDD and migraine via HPA-axis hyperactivity (23), which specifically characterizes melancholic depression (24). However, findings regarding a potential association between HPA-axis dysregulation and migraine are still limited and controversial (25). Another mechanism potentially implicated in the association between melancholic MDD and migraine is a strong activation of the noradrenergic system in the central nervous system. This hypothesis is supported by the observation that a hypernoradrenergic state has been associated with melancholic depression (26), while a recent preclinical study provided evidence for a complex role of locus ceruleus dysregulation in two key mechanisms putatively underlying migraine; that is, cortical-spreading depression and trigeminovascular activation (27). Although based on only very few data hitherto available in the literature, it may be worthwhile to further study the role of dysfunctional locus coeruleus in migraine pathogenesis and the emergence of comorbid depression and migraine.

Disturbances in sleep and appetite observed in melancholic depression may also share molecular mechanisms with migraine pathophysiology. For instance, melatonin MT1 receptors have been recently implicated in the pathogenesis of melancholic depression (28). Melatonin, a hormone primarily involved in the regulation of the sleep-wake cycle, might interact with calcitonin gene-related peptide (CGRP) effects. Specifically, melatonin was shown to inhibit CGRPinduced vasodilation of cerebral arteries in rats (29), as well as to downregulate CGRP mRNA levels in human peripheral blood mononuclear cells from migraine patients (30). CGRP levels are increased in migraine patients both during attacks and in the interictal state, compared to controls (31), and CGRP has been suggested to regulate appetite and energy intake (32). Interestingly, melatonin has been used as an oral 
Table 4. Association between lifetime MDD subtypes and migraine frequency (number of migraine days per month).

\begin{tabular}{|c|c|c|c|c|c|c|c|c|c|}
\hline & \multicolumn{3}{|l|}{ Any migraine $(n=428)$} & \multicolumn{3}{|c|}{ Migraine without aura $(\mathrm{n}=284)$} & \multicolumn{3}{|c|}{ Migraine with aura $(n=144)$} \\
\hline & $\begin{array}{l}\text { Migraine days per } \\
\text { month (mean } \pm S D)\end{array}$ & $\beta$ & $p$ & $\begin{array}{l}\text { Migraine days per } \\
\text { month (mean } \pm S D \text { ) }\end{array}$ & $\beta$ & $p$ & $\begin{array}{l}\text { Migraine days per } \\
\text { month (mean } \pm S D)\end{array}$ & $\beta$ & $p$ \\
\hline Lifetime MDD, any & $3.12( \pm 5.0)$ & 0.09 & 0.053 & $3.05( \pm 4.8)$ & $-^{2}$ & $-{ }^{2}$ & $3.24( \pm 5.3)$ & -2 & $-{ }^{2}$ \\
\hline Melancholic MDD & $2.61( \pm 3.8)$ & -1 & -1 & $2.16( \pm 2.7)$ & 0.01 & 0.924 & $3.44( \pm 5.3)$ & 0.18 & 0.050 \\
\hline Atypical MDD & $4.00( \pm 7.2)$ & 0.03 & 0.558 & $3.85( \pm 6.7)$ & $-^{2}$ & $-{ }^{2}$ & $4.45( \pm 9.1)$ & $-{ }^{2}$ & $-{ }^{2}$ \\
\hline Combined MDD & $5.47( \pm 8.2)$ & 0.12 & 0.019 & $5.82( \pm 8.2)$ & $-^{2}$ & $-{ }^{2}$ & $5.08( \pm 8.1)$ & $-{ }^{2}$ & $-{ }^{2}$ \\
\hline Unspecified MDD & $2.64( \pm 3.3)$ & 0.07 & 0.194 & $2.92( \pm 3.8)$ & $-{ }^{2}$ & $-{ }^{2}$ & $2.24( \pm 2.5)$ & $-{ }^{2}$ & $-{ }^{2}$ \\
\hline No MDD & $2.29( \pm 3.7)$ & Refer & ence & $2.48( \pm 4.0)$ & Refer & nce & $1.82( \pm 2.6)$ & Refer & ence \\
\hline
\end{tabular}

MDD: major depressive disorder; SD: standard deviation.

$\beta$ : according to linear regression with adjustment for age, sex, socioeconomic status.

IIn presence of significant interactions, only results for migraine subtypes are reported.

${ }^{2}$ In the absence of significant interactions, only results for any migraine are reported.

Note: Significant results after correction for multiple testing in bold.

Table 5. Associations between lifetime MDD subtypes, migraine subtypes and duration (hours) of migraine attacks.

\begin{tabular}{|c|c|c|c|c|c|c|c|c|c|}
\hline & \multicolumn{3}{|c|}{ Any migraine $(n=442)$} & \multicolumn{3}{|c|}{ Migraine without aura $(n=294)$} & \multicolumn{3}{|c|}{ Migraine with aura $(n=148)$} \\
\hline & $\begin{array}{l}\text { Attack duration } \\
\text { (mean } \pm S D)\end{array}$ & $\beta$ & $p$ & $\begin{array}{l}\text { Attack duration } \\
\text { (mean } \pm S D)\end{array}$ & $\beta$ & $p$ & $\begin{array}{l}\text { Attack duration } \\
\text { (mean } \pm S D)\end{array}$ & $\beta$ & $p$ \\
\hline MDD (any) & $28.43( \pm 24.4)$ & -1 & -1 & $30.31( \pm 23.6)$ & 0.00 & 0.997 & $25.20( \pm 25.5)$ & 0.18 & 0.029 \\
\hline Melancholic MDD & $31.32( \pm 25.3)$ & -1 & -1 & $32.16( \pm 24.3)$ & 0.01 & 0.821 & $29.8 \mathrm{I}( \pm 27.2)$ & 0.20 & 0.028 \\
\hline Atypical MDD & $24.08( \pm 20.9)$ & 0.01 & 0.864 & $29.07( \pm 20.7)$ & $-{ }^{2}$ & $-{ }^{2}$ & $9.60( \pm 13.8)$ & $-{ }^{2}$ & $-^{2}$ \\
\hline Combined MDD & $30.35( \pm 31.3)$ & -1 & -1 & $26.92( \pm 28.6)$ & -0.07 & 0.266 & $33.77( \pm 34.6)$ & 0.14 & 0.095 \\
\hline Unspecified MDD & $26.98( \pm 22.7)$ & 0.00 & 0.998 & $29.86( \pm 23.4)$ & $-^{2}$ & $-^{2}$ & $22.50( \pm 21.1)$ & $-{ }^{2}$ & $--^{2}$ \\
\hline No MDD & $24.70( \pm 22.9)$ & Refer & ence & $27.96( \pm 23.4)$ & Refere & & $16.7 \mid( \pm$ 19.6) & Refer & nce \\
\hline
\end{tabular}

MDD: major depressive disorder; SD: standard deviation.

$\beta$ : according to linear regression with adjustment for age, sex, socioeconomic status.

'In the presence of significant interactions, only results for migraine subtypes are reported.

${ }^{2}$ In the absence of significant interactions, only results for any migraine are reported.

Note: No results were significant after multiple testing correction.

supplement for migraine prophylaxis, although the number of studies examining its efficacy is still limited (33). Hence, melatonin may be involved in some of the shared features of melancholic depression and migraine that relate to disturbances of sleep and appetite.

Our results showed lifetime MDD to be associated with a higher migraine intensity exclusively in the group of participants with MWA. Our findings fit to the earlier formulated hypothesis that depression might be more strongly correlated with MWA compared to MWOA (34). The association we observed might be mediated by mechanisms specifically associated with MWA, such as brain hypoperfusion (35), as more severe and spread patterns of brain hypoperfusion disorders have been shown in subjects with either melancholic or unspecified MDD compared to atypical MDD (36). These findings, together with our observation of an association of unspecified MDD with increased migraine intensity, support the need for future studies to specifically evaluate associations between depression subtypes and migraine severity. Additionally, we found the combined MDD subtype (which includes participants with both melancholic and atypical characteristics) to be associated with higher migraine frequency independent of migraine subtype. Our results are in accordance with a recent study showing an association between depression symptoms and migraine frequency in patients with both migraine with and without aura (37). However, no information on MDD subtypes was available in this study.

Our observation of differential associations between the subtypes of MDD and migraine supports the need for studies aiming to investigate the specific mechanisms that could underlie the associations between 
MDD subtypes and migraine subtypes. For instance, since the existence of shared genetic determinants between MDD and migraine has been suggested (38), cross-trait analyses with a specific focus on melancholic MDD and migraine might be of help in understanding whether specific genetic variants might predispose to developing both conditions. Migraine subtype might also play an important role in this relationship, based on the hypothesis of a different genetic background between these two forms of migraine (39). These studies might also help to understand whether patients with comorbid melancholic depression might represent a migraine subphenotype. Based on our observation of nominal associations between melancholic but not atypical depression and migraine severity, it might be speculated that these patients might benefit from tailored treatment strategies, also based on the fact that these two MDD subtypes have been suggested to differ in response to antidepressants (20). Future studies might also elucidate whether melatonin, which is currently being investigated as a promising option to treat both migraine and melancholic MDD, might be particularly useful in patients with both disorders.

The results of our study need to be viewed in light of some limitations. First, our data were cross-sectional and did not allow us to determine the direction of the association between MDD/MDD subtypes and migraine severity given that severe migraine could also increase the risk of MDD. Hence, longitudinal studies are required to determine the time sequence between the onset of MDD and migraine. Second, our semi-structured interviews elicited criteria for MDD and migraine across the lifetime, entailing the risk of inaccurate recall of remote episodes. Third, migraine intensity was assessed using a categorical ("light", "moderate" or "severe") rather than a continuous approach. Fourth, migraine diagnoses relied on interviews conducted by psychologists rather than headache-trained physicians. Fifth, the limited number of subjects with the atypical or combined MDD subtypes might have limited our power to identify associations with small effect sizes within models stratified according to migraine subtype.

\section{Conclusion}

Our data provide evidence for divergent associations of lifetime MDD and MDD subtypes with the prevalence and severity of migraine in subjects with MWOA or MWA, emphasizing the need for subtyping participants according to MDD and migraine subtypes in future studies. Further studies exploring the pathophysiological mechanisms shared between melancholic depression and migraine are warranted.

\section{Clinical implications}

- Melancholic, combined and unspecified major depressive disorder (MDD) were associated with increased frequency of migraine with aura, whereas only melancholic MDD was associated with increased frequency of migraine without aura.

- Lifetime MDD and the unspecified subtype were associated with increased migraine severity exclusively in subjects with migraine with aura.

\section{Acknowledgements}

The authors thank Lausanne inhabitants who volunteered to participate in the CoLaus|PsyCoLaus study, Professor Peter Vollenweider, Professor Gérard Waeber, and the collaborators who contributed to the coordination of the study and the collection of data.

\section{Declaration of conflicting interests}

The authors declared no potential conflicts of interest with respect to the research, authorship, and/or publication of this article.

\section{Funding}

The authors disclosed receipt of the following financial support for the research, authorship, and/or publication of this article: The CoLaus|PsyCoLaus study was and is supported by research grants from GlaxoSmithKline, the Faculty of
Biology and Medicine of Lausanne, and the Swiss National Science Foundation (grants 3200B0-105993, 3200B0-118308, 33CSCO-122661, 33CS30-139468 and 33CS30-148401). This study was supported by a grant from the Svenska Läkaresällskapet (SLS) to JM, by a grant from the Swedish Research Council to HBS, and in part by the Intramural Research Program of the National Institute of Mental Health (ZIAMH002932). Claudia Pisanu is supported by a fellowship financed by Fondazione Umberto Veronesi. JG received support from the Swiss National Science Foundation and from the Intramural Research Program of the National Institute of Mental Health through a Visiting Fellowship.

\section{References}

1. Breslau N, Schultz LR, Stewart WF, et al. Headache and major depression: Is the association specific to migraine? Neurology 2000; 54: 308-313. 
2. Levinson DF. The genetics of depression: A review. Biological Psychiatry 2006; 60: 84-92.

3. Goulart AC, Santos IS, Brunoni AR, et al. Migraine headaches and mood/anxiety disorders in the ELSA Brazil. Headache 2014; 54: 1310-1319.

4. Yang Y, Zhao H, Heath AC, et al. Familial aggregation of migraine and depression: Insights from a large Australian twin sample. Twin Res Human Genet 2016; 19: 312-321.

5. Ashina S, Serrano D, Lipton RB, et al. Depression and risk of transformation of episodic to chronic migraine. $J$ Headache Pain 2012; 13: 615-624.

6. Musliner KL, Munk-Olsen T, Laursen TM, et al. Heterogeneity in 10-year course trajectories of moderate to severe major depressive disorder: A Danish National Register-based study. JAMA Psychiatry 2016; 73: 346-353.

7. Rudaz DA, Vandeleur CL, Gebreab SZ, et al. Partially distinct combinations of psychological, metabolic and inflammatory risk factors are prospectively associated with the onset of the subtypes of major depressive disorder in midlife. $J$ Affective Dis 2017; 222: 195-203.

8. Lamers F, de Jonge P, Nolen WA, et al. Identifying depressive subtypes in a large cohort study: Results from the Netherlands Study of Depression and Anxiety (NESDA). J Clinical Psych 2010; 71: 1582-1589.

9. Penninx BW, Milaneschi Y, Lamers F, et al. Understanding the somatic consequences of depression: Biological mechanisms and the role of depression symptom profile. BMC Medicine 2013; 11: 129.

10. Glaus J, von Kanel R, Lasserre AM, et al. Mood disorders and circulating levels of inflammatory markers in a longitudinal population-based study. Psychol Med 2018; 48: 961-973.

11. Firmann M, Mayor V, Vidal PM, et al. The CoLaus study: A population-based study to investigate the epidemiology and genetic determinants of cardiovascular risk factors and metabolic syndrome. BMC Cardiovasc Dis 2008; 8: 6.

12. Preisig M, Waeber G, Vollenweider P, et al. The PsyCoLaus study: Methodology and characteristics of the sample of a population-based survey on psychiatric disorders and their association with genetic and cardiovascular risk factors. BMC Psychiatry 2009; 9: 9.

13. Leboyer M, Barbe B, Gorwood P, et al. Interview Diagnostique Pour Les Études Génétiques. 1995.

14. Nurnberger JI Jr, Blehar MC, Kaufmann CA, et al. Diagnostic interview for genetic studies. Rationale, unique features, and training. NIMH Genetics Initiative. Arch Gen Psychiatry 1994; 51: 849-859; discussion 863-844.

15. Headache Classification Subcommittee of the International Headache Society. The International Classification of Headache Disorders, 2nd edition. Cephalalgia 2004; 24: 9-160.

16. Angst J, Gamma A, Benazzi F, et al. Atypical depressive syndromes in varying definitions. Euro Arch Psych Clinical Neurosci 2006; 256: 44-54.

17. Hollingshead AA. Four-factor index of social status. New Haven, CT: Yale University, 1975.
18. Yang Y, Zhao H, Heath AC, et al. Shared genetic factors underlie migraine and depression. Twin Res Hum Genet 2016; 19: 341-350.

19. Laurell K, Artto V, Bendtsen L, et al. Premonitory symptoms in migraine: A cross-sectional study in 2714 persons. Cephalalgia 2016; 36: 951-959.

20. Lojko D and Rybakowski JK. Atypical depression: Current perspectives. Neuropsych Dis Treatment 2017; 13: 2447-2456.

21. Fasick V, Spengler RN, Samankan S, et al. The hippocampus and TNF: Common links between chronic pain and depression. Neurosci Biobehavioral Rev 2015; 53: 139-159.

22. Liu HY, Chou KH and Chen WT. Migraine and the hippocampus. Curr Pain Headache Rep 2018; 22: 13.

23. Zhu LJ, Liu MY, Li H, et al. The different roles of glucocorticoids in the hippocampus and hypothalamus in chronic stress-induced HPA axis hyperactivity. PloS One 2014; 9: e97689.

24. Juruena MF, Bocharova M, Agustini B, et al. Atypical depression and non-atypical depression: Is HPA axis function a biomarker? A systematic review. I Affective Dis 2018; 233: 45-67.

25. Lippi G and Mattiuzzi C. Cortisol and migraine: A systematic literature review. Agri 2017; 29: 95-99.

26. Gold PW. The organization of the stress system and its dysregulation in depressive illness. Mol Psych 2015; 20: 32-47.

27. Vila-Pueyo M, Strother LC, Kefel M, et al. Divergent influences of the locus coeruleus on migraine pathophysiology. Pain 2018; 160: 385-394.

28. Comai S, Ochoa-Sanchez R, Dominguez-Lopez S, et al. Melancholic-like behaviors and circadian neurobiological abnormalities in melatonin MT1 receptor knockout mice. Int J Neuropsych 2015; 18: pyu075.

29. Viswanathan M. Melatonin inhibits calcitonin generelated peptide-induced vasodilation and increase in cAMP in rat middle cerebral arteries. Euro $J$ Pharmacol 2001; 415: 247-250.

30. Ansari M, Karkhaneh A, Kheirollahi A, et al. The effect of melatonin on gene expression of calcitonin generelated peptide and some proinflammatory mediators in patients with pure menstrual migraine. Acta Neurologica Belgica 2017; 117: 677-685.

31. Kamm K, Straube A and Ruscheweyh R. Calcitonin gene-related peptide levels in tear fluid are elevated in migraine patients compared to healthy controls. Cephalalgia 2019; 39: 1535-1543.

32. Sanford D, Luong L, Gabalski A, et al. An intraperitoneal treatment with calcitonin gene-related peptide (CGRP) regulates appetite, energy intake/expenditure, and metabolism. J Molecular Neurosci 2019; 67: 28-37.

33. Long R, Zhu Y and Zhou S. Therapeutic role of melatonin in migraine prophylaxis: A systematic review. Medicine (Baltimore) 2019; 98: e14099.

34. Ligthart L, Penninx BW, Nyholt DR, et al. Migraine symptomatology and major depressive disorder. Cephalalgia 2010; 30: 1073-1081.

35. Charles A. The migraine aura. Continuum (Minneap Minn) 2018; 24: 1009-1022. 
36. Fountoulakis KN, Iacovides A, Gerasimou G, et al. The relationship of regional cerebral blood flow with subtypes of major depression. Progress Neuropsychopharmacol Biol Psych 2004; 28: 537-546.

37. Szabo E, Galambos A, Kocsel N, et al. Association between migraine frequency and neural response to emotional faces: An fMRI study. NeuroImage Clin 2019; 22: 101790 .
38. Yang Y, Zhao H, Boomsma DI, et al. Molecular genetic overlap between migraine and major depressive disorder. Euro J Hum Genet 2018; 26: 1202-1216.

39. Pisanu C, Preisig M, Castelao E, et al. A genetic risk score is differentially associated with migraine with and without aura. Hum Genet 2017; 136: 999-1008. 